\title{
Qualidade de vida em homens usuários de Unidade de Saúde da Família
}

\author{
Quality of life among male patients of a Family Health Unit
}

Luiz Gustavo Silva Souzaa, Ana Claudia Ferreira Sanches ${ }^{b}$, Alexsandro Luiz de Andrade ${ }^{c}$

a Psicólogo. Doutor em Psicologia, Universidade Federal Fluminense.

b Psicóloga. Mestre em Psicologia, Universidade Federal do Espírito Santo.

c Psicólogo. Doutor em Psicologia, Universidade Federal do Espírito Santo.

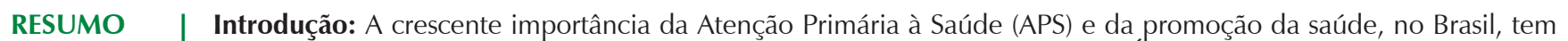
colocado em evidência o tópico da qualidade de vida de usuários do Sistema Único de Saúde (SUS).

Objetivo: Correlacionar a qualidade de vida (QV) de homens de classe popular, usuários de Unidade de Saúde da Família (USF), com variáveis sociodemográficas.

Materiais e Métodos: Estudo transversal analítico. Participaram 300 homens, usuários de uma USF do município de Vitória, ES. Eles responderam ao WHOQOL-BREF, aplicado como entrevista individual, e a um questionário sociodemográfico. Os dados foram analisados com teste t, ANOVA, ANCOVA e coeficiente de correlação de Pearson. Resultados: Os usuários que estavam trabalhando obtiveram escores maiores do que aqueles que não estavam trabalhando nos domínios de QV: global $(t=1,98 ; p=0,04)$, físico $(t=4,88 ; p<0,001)$, psicológico $(t=2,46 ; p=0,01)$ e social $(t=2,89 ; p=0,004)$. A idade apresentou correlação negativa com os domínios de QV: global $(r=-0,11$; $p<0,05)$, físico $(r=-0,22 ; p<0,01)$, psicológico $(r=-0,12 ; p<0,05)$ e social $(r=-0,15 ; p<0,01)$. A renda apresentou correlação positiva com todos os domínios de QV: global $(r=0,19 ; p<0,01)$, físico $(r=0,22 ; p<0,01)$, psicológico $(r=0,14 ; p<0,05)$, social $(r=0,16 ; p<0,01)$ e ambiental $(r=0,32 ; p<0,01)$. A escolaridade apresentou correlação positiva com os domínios físico $(r=0,13 ; p<0,05)$, social $(r=0,15, p<0,01)$ e ambiental $(r=0,19 ; p<0,01)$.

Conclusão: Foi identificada relação de maiores níveis percebidos de QV em homens de classe popular que tinham trabalho, menor idade e maior escolaridade e renda.

Palavras-chave: qualidade de vida; saúde do homem; atenção primária à saúde.

\begin{tabular}{l|l} 
ABSTRACT & Introduction: The growing importance of Primary Health Care (PHC) and of Health Promotion in Brazil highlights the topic of quality
\end{tabular} of life among patients of the Brazilian Health System.

Objective: To correlate the quality of life (QL) to sociodemographic variables in low-income male patients of Brazilian PHC.

Materials and Methods: Cross-sectional analytical study. Three hundred male patients from a PHC service in Vitória, ES, Brazil, participated in this research. They answered to the WHOQOL-BREF administered in individual interviews, and to a sociodemographic questionnaire. We analyzed the data with t-test, ANOVA, ANCOVA and with Pearson correlation coefficient.

Results: Patients who were working had higher scores than patients who were not working in the following QL domains: global $(t=1.98 ; p=0.04)$, physical $(t=4.88 ; p<0.001)$, psychological $(t=2.46 ; p=0.01)$ and social $(t=2.89 ; p=0.004)$. Age was negatively correlated with the following QL domains: global $(r=-0.11 ; p<0.05)$, physical $(r=-0.22 ; p<0.01)$, psychological $(r=-0.12 ; p<0.05)$ and social $(r=-0.15 ; p<0.01)$. Income was positively correlated with all QL domains: global $(r=0.19 ; p<0.01)$, physical $(r=0.22$; $p<0.01)$, psychological $(r=0.14 ; p<0.05)$, social $(r=0.16 ; p<0.01)$ and environmental $(r=0.32 ; p<0.01)$. Education was positively correlated with the physical $(r=0.13 ; p<0.05)$, social $(r=0.15 ; p<0.01)$ and environmental $(r=0.19 ; p<0.01)$ domains.

Conclusion: Among the low-income male patients of Brazilian PHC, greater levels of perceived QL were related to having a job, lower age, more education and greater income.

Keywords: quality of life; men's health; primary health care. 


\section{INTRODUÇÃO}

A Atenção Primária à Saúde (APS) ganhou crescente importância nas últimas décadas, em âmbito nacional e internacional. No Brasil, a Estratégia Saúde da Família foi apresentada, na década de 1990, como estratégia para reorientação da APS e de todo o sistema de saúde brasileiro, em direção à efetivação das diretrizes do Sistema Único de Saúde (SUS), como a universalidade e a integralidade, e à superação do chamado modelo biomédico ${ }^{1,2}$. Políticas e práticas de saúde com foco na promoção de saúde foram propostas para favorecer a qualidade de vida de sujeitos, grupos e populações, contribuindo para a prevenção de doenças.

Qualidade de vida (QV) é um constructo de difícil conceituação e há diferentes definições na literatura. $\mathrm{O}$ interesse por esse fenômeno conheceu pronunciado crescimento nas últimas décadas do século $\mathrm{XX}$, o que está ligado ao redirecionamento das políticas de saúde para ação sobre estilos de vida. O conceito de QV foi e é importante para superar visões e práticas de saúde excessivamente centradas nos aspectos biomédicos e que desconsideram aspectos sociais, culturais e psicológicos ${ }^{3}$.

Apesar das divergências nas definições, há relativo consenso sobre duas características da QV, sua dimensão subjetiva e sua multidimensionalidade ${ }^{3,4}$. Essas características embasam a definição proposta pela Organização Mundial da Saúde (OMS) tal como se segue: "a percepção do indivíduo sobre a sua posição na vida, no contexto da cultura e dos sistemas de valores nos quais ele vive, e em relação a seus objetivos, expectativas, padrões e preocupações"4:1405.

Trata-se de uma "definição combinada" de QV³, que considera o aspecto global, visto como a medida de satisfação com a vida em geral, bem como possíveis dimensões que compõem a QV. Foram desenvolvidos diferentes instrumentos para avaliação da QV condizentes com essa definição, dentre eles um instrumento abreviado, o Whoqol-Bref, um dos mais utilizados em pesquisas sobre o assunto, amplamente testado e com propriedades psicométricas adequadas de validade e precisão $0^{5,6}$.

Diversas pesquisas mostraram a associação de morbidades, variáveis sociodemográficas e percepção de QV. Um estudo com usuários de APS portadores de doenças crônicas mostrou que essa condição está associada a percepções menos favoráveis sobre a QV. Mostrou ainda que a percepção dos diversos domínios de QV era menos favorável em usuários com mais idade e com classe socioeconômica mais baixa ${ }^{7}$. Correlações negativas entre domínios de QV e idade foram encontradas em usuários com doença renal crônica ${ }^{8,9}$. Resultados de Lara et al. ${ }^{9}$ indicam que o tratamento fisioterapêutico possui impacto positivo na QV desses usuários.
Blay \& Marchesoni ${ }^{6}$ investigaram se morbidades psiquiátricas levariam à percepção menos favorável sobre QV, o que foi, em geral, confirmado. Encontraram ainda correlação positiva entre a renda dos participantes e a percepção mais favorável do domínio ambiental de QV. A associação entre maior renda e melhor percepção do domínio ambiental também foi verificada em idosos investigados por Alexandre et al. ${ }^{10}$.

A consideração da QV mostra-se relevante também para as políticas e práticas de saúde dirigidas especificamente à população masculina. Um dos eixos da Política Nacional de Atenção Integral à Saúde do Homem ${ }^{11}$ afirma a necessidade de privilegiar "um novo paradigma baseado na atenção integral, valorizando, fundamentalmente, a promoção da saúde e a qualidade de vida". Essa Política postula o alinhamento com as diretrizes da Atenção Básica e propõe que se deve favorecer a transformação de estilos de vida e a construção de práticas de prevenção de doenças e de promoção de saúde junto aos usuários. Esses objetivos são especialmente difíceis no caso dos homens, tendo em vista tradicionais estereótipos de gênero que, tanto da parte dos usuários quanto dos próprios serviços de saúde, afastam os homens do cuidado e do autocuidado ${ }^{11,12,13}$.

Pesquisas demonstraram que crenças e práticas de saúde são formas de expressão do gênero, formas construídas culturalmente de pensar e viver a feminilidade e a masculinidade, suas dinâmicas relacionais e de poder. O estereótipo da masculinidade, em sua versão mais difundida, contribui para a construção de relações problemáticas entre homens e práticas de saúde. Afirmar a masculinidade corresponde frequentemente a desprezar o autocuidado físico, emocional, psicológico e social. O trabalho pago é visto como aspecto central da definição de si e da autoestima, colocando-se hegemonicamente acima das necessidades de saúde ${ }^{12}$.

Nesse contexto, a presente pesquisa teve o objetivo de correlacionar a QV de homens de classe popular, usuários de Unidade de Saúde da Família, com variáveis sociodemográficas. Procurou, com isso, contribuir para a literatura científica brasileira sobre QV, gerando dados e discussões específicos sobre QV em homens de classe popular. Buscou promover ainda reflexões sobre QV que considerem aspectos da produção cultural da masculinidade e fornecer subsídios para práticas de cuidado dirigidas a homens em Atenção Primária à Saúde.

\section{MATERIAIS E MÉTODOS}

Trata-se de estudo transversal e analítico. Participaram 300 homens adultos, usuários de uma Unidade de Saúde da Família (USF) do município de Vitória, ES. Os critérios de inclusão foram os seguintes: ter 18 anos de idade ou mais, ter 
se dirigido à USF para buscar atendimento, ter capacidade de responder individualmente aos instrumentos de pesquisa mediante entrevista, ter concordado com a participação voluntária na pesquisa.

Os participantes foram recrutados por conveniência, dentro da USF, durante seu horário regular de funcionamento, nos meses de fevereiro a julho de 2013. Após aceitação do convite para participação, foram dirigidos para uma sala reservada, na qual responderam individualmente aos instrumentos de pesquisa, que foram aplicados no formato de entrevista (heteroaplicação). Esse formato foi escolhido para garantir o melhor entendimento das questões que compunham os instrumentos, pois muitos participantes, com baixa escolaridade, teriam dificuldades com a autoaplicação.

Foram utilizados um questionário sociodemográfico e a versão em português do Whoqol-Bref ${ }^{5}$. O Whoqol-Bref contém duas questões sobre percepção global de QV e outras 24 questões para quatro domínios específicos: físico, psicológico, social e ambiental. Cada uma das questões adicionais corresponde a uma faceta da QV, integrando as 24 facetas que emergiram do processo de definição do construto. Trata-se de aspectos tais como "dor e desconforto", "energia e fadiga" (domínio físico); "autoestima", "imagem corporal e aparência" (domínio psicológico); "relações pessoais", "suporte social" (domínio de relações sociais) e "segurança física e proteção", "ambiente no lar" (domínio ambiental)5.

Os resultados do Whoqol-Bref foram convertidos em escala de 0 a 100. Na interpretação da medida, os níveis de QV são menores quanto mais próximos de 0 (zero) e maiores quanto mais próximos de 100 (cem). Os dados foram analisados com auxílio do software SPSS, versão 18.0, e foram utilizadas as seguintes técnicas: estatísticas descritivas (frequência, média, mínimo, máximo, desvio padrão); teste t para amostras independentes; análises de variância (ANOVA, ANCOVA) e coeficiente de correlação de Pearson. O valor $p<0,05$ foi considerado como nível de significância estatística.

Os preceitos de ética em pesquisa com seres humanos foram respeitados. Todos os participantes assinaram Termo de Consentimento Livre e Esclarecido. Foram seguidas as recomendações do Conselho Nacional de Saúde (Resolução CNS 466/2012). O projeto foi aprovado pelo Comitê de Ética em Pesquisa da Universidade Federal do Espírito Santo, com o parecer número 233.306.

\section{RESULTADOS}

Os homens investigados $(n=300)$ apresentaram média de idade de 43,14 15,05 anos, com mínimo de 18 e máximo de 84 anos de idade. A média da renda familiar mensal per capita foi de $R \$ 652,69 \pm 477,61$. O valor médio da renda familiar mensal total foi de $\mathrm{R} \$ 1.898,25 \pm 1.319,44$, o que caracte- riza os usuários investigados em geral como "Classe C" (C1, C2), de acordo com o Critério Brasil ${ }^{14}$. A Tabela 1 relaciona as demais características sociodemográficas dos participantes.

Tabela 1. Caracterização sociodemográfica dos usuários investigados $(n=300)$.

\begin{tabular}{|c|c|c|}
\hline & $\mathbf{n}$ & $\%$ \\
\hline \multicolumn{3}{|l|}{ Escolaridade } \\
\hline Sem escolaridade & 11 & 3,66 \\
\hline Ensino Fundamental incompleto & 110 & 36,66 \\
\hline Ensino Fundamental completo & 30 & 10,00 \\
\hline Ensino Médio incompleto & 51 & 17,00 \\
\hline Ensino Médio completo & 73 & 24,33 \\
\hline Ensino Superior incompleto & 10 & 3,33 \\
\hline Ensino Superior completo & 15 & 5,00 \\
\hline \multicolumn{3}{|l|}{ Situação de trabalho } \\
\hline Desempregado & 44 & 14,66 \\
\hline Sem carteira assinada ou autônomo & 60 & 20,00 \\
\hline Com carteira assinada & 139 & 46,33 \\
\hline Aposentado & 51 & 17,00 \\
\hline Outra & 6 & 2,00 \\
\hline \multicolumn{3}{|l|}{ Atualmente trabalhando } \\
\hline Sim & 200 & 66,66 \\
\hline Não (desempregados, aposentados e outros) & 100 & 33,33 \\
\hline \multicolumn{3}{|l|}{ Possui plano privado de saúde } \\
\hline Sim & 62 & 20,66 \\
\hline Não & 238 & 79,33 \\
\hline \multicolumn{3}{|l|}{ Estado civil } \\
\hline Solteiro & 67 & 22,33 \\
\hline Casado & 143 & 47,66 \\
\hline União estável & 70 & 23,33 \\
\hline Divorciado & 14 & 4,66 \\
\hline Viúvo & 6 & 2,00 \\
\hline \multicolumn{3}{|l|}{ Tem filhos } \\
\hline Sim & 237 & 79,00 \\
\hline Não & 63 & 21,00 \\
\hline \multicolumn{3}{|l|}{ Tem religião } \\
\hline Sim & 240 & 80,00 \\
\hline Não & 60 & 20,00 \\
\hline \multicolumn{3}{|l|}{ Considera-se praticante da religião $(n=240)$} \\
\hline Sim & 150 & 62,50 \\
\hline Não & 90 & 37,50 \\
\hline \multicolumn{3}{|l|}{ Designação religiosa } \\
\hline Evangélica & 125 & 41,66 \\
\hline Católica & 112 & 37,33 \\
\hline Outras & 63 & 21,00 \\
\hline
\end{tabular}

A escolaridade mais frequente foi o Ensino Fundamental incompleto (36,66\%). Do grupo geral, nota-se que $67,32 \%$ não possuíam Ensino Médio completo. A maioria dos participantes possuía trabalho com ou sem carteira assinada (66,33\%). Declararam-se aposentados 17,00\% e sem trabalho 14,66\%. Com relação à questão sobre estar trabalhando atualmente, $66,66 \%$ responderam que sim e $33,33 \%$ responderam que não. Cerca de oito em cada dez entrevistados não possuía plano privado de saúde, o que confirma a importância dos serviços públicos de saúde para a população investigada. 
Declararam-se casados ou em união estável 71,00\% e 79,00\% tinham filhos. A maioria declarou possuir religião $(80,00 \%)$ e, dentre esses, $62,50 \%$ consideravam-se praticantes.

Os resultados do Whoqol-Bref, para percepção sobre QV, foram convertidos em escala de 0 a 100 . Foram verificadas as seguintes médias: percepção global $(60,12 \pm 22,49)$, domínio físico $(68,58 \pm 18,91)$, domínio psicológico $(74,88 \pm 15,71)$, domínio social $(65,69 \pm 22,98)$, e domínio ambiental $(57,96 \pm 17,53)$.

O teste t para amostras independentes foi utilizado para comparar os escores médios dos domínios de QV entre subgrupos de participantes. A QV global foi maior entre os que estavam trabalhando do que entre aqueles que não estavam (desempregados, aposentados e outros) e essa diferença mostrou-se estatisticamente significativa, assim como para todos os outros domínios de QV exceto para o domínio ambiental, como mostra a Tabela 2.

Tabela 2. Comparação de escores médios de qualidade de vida entre homens que estavam trabalhando $(n=200)$ e aqueles que não estavam $(n=100)$.

\begin{tabular}{lccccc}
\hline $\begin{array}{c}\text { Domínios } \\
\text { de qualidade } \\
\text { de vida }\end{array}$ & $\begin{array}{c}\text { Com trabalho } \\
\boldsymbol{M} \pm \boldsymbol{D} \boldsymbol{P}\end{array}$ & $\begin{array}{c}\text { Sem trabalho } \\
\boldsymbol{M} \pm \boldsymbol{D P}\end{array}$ & $\boldsymbol{t}$ & $\boldsymbol{d f}$ & $\boldsymbol{p}$ \\
\hline Global & $61,93 \pm 21,27$ & $56,50 \pm 24,45$ & 1,98 & 298 & 0,048 \\
Físico & $72,41 \pm 17,03$ & $60,92 \pm 20,20$ & 4,88 & 298 & $<0,001$ \\
Psicológico & $76,45 \pm 14,85$ & $71,75 \pm 16,95$ & 2,46 & 298 & 0,014 \\
Social & $68,37 \pm 21,72$ & $60,33 \pm 24,56$ & 2,89 & 298 & 0,004 \\
Ambiental & $58,54 \pm 17,18$ & $56,81 \pm 18,23$ & 0,80 & 298 & 0,420 \\
\hline
\end{tabular}

A constatação das diferenças encontradas na QV de participantes que estavam ou não trabalhando levou a um questionamento sobre a possível influência das covariáveis idade e renda. Os aposentados foram incluídos na categoria "sem trabalho" e possuíam média de idade $(63,02 \pm 10,43)$ superior à média geral $(43,14 \pm 15,05)$. Aqueles que estavam trabalhando possuíam renda familiar mensal maior que aqueles que não estavam trabalhando $(687,21 \pm 488,56$ versus $583,66 \pm 449,38)$.

Uma ANCOVA foi conduzida para avaliar diferenças na QV entre aqueles que estavam trabalhando e aqueles que não estavam, controlando idade e renda. Registrou-se diferença no domínio físico, com as seguintes médias ajustadas: $71,25 \pm 1,28$ para aqueles que estavam trabalhando e $63,24 \pm 1,87$ para aqueles que não estavam trabalhando, $[F(1,296)=11,46, p=0,001]$.

Possuir ou não plano privado de saúde não esteve associado a diferenças estatisticamente significativas em qualquer domínio de QV. O mesmo foi verificado para ter ou não ter relação afetiva estável (casados e homens em união estável versus solteiros, divorciados e viúvos); ter ou não ter filhos; ter ou não ter religião; declarar-se praticante ou não praticante da religião. Uma ANOVA constatou igualmente ausência de diferença estatisticamente significativa entre designações religiosas.

A Tabela 3 apresenta resultados de correlação entre as variáveis idade, renda familiar mensal per capita, escolaridade e domínios de QV. Para essa análise, os níveis

Tabela 3. Média, desvio padrão e análise de correlação das variáveis idade, renda familiar mensal per capita, escolaridade e domínios de qualidade de vida $(n=300)$.

\begin{tabular}{|c|c|c|c|c|c|c|c|c|c|c|}
\hline \multirow{5}{*}{ 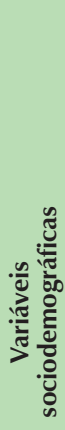 } & \multirow{4}{*}{$\begin{array}{l}\text { Idade } \\
\text { Renda familiar } \\
\text { mensal per capita }\end{array}$} & \multirow[b]{2}{*}{$\mathrm{M} \pm \mathrm{DP}$} & \multicolumn{3}{|c|}{$\begin{array}{c}\text { Variáveis } \\
\text { sociodemográficas }\end{array}$} & \multicolumn{5}{|c|}{ Domínios da QV } \\
\hline & & & Idade & $\begin{array}{c}\text { Renda } \\
\text { familiar } \\
\text { mensal } \\
\text { per capita }\end{array}$ & Escolaridade & $\begin{array}{c}\text { Domínio } \\
\text { físico de QV }\end{array}$ & $\begin{array}{l}\text { Domínio } \\
\text { psicológico } \\
\text { de QV }\end{array}$ & $\begin{array}{c}\text { Domínio } \\
\text { social de } \\
\text { QV }\end{array}$ & $\begin{array}{c}\text { Domínio } \\
\text { ambiental } \\
\text { de QV }\end{array}$ & $\begin{array}{c}\text { Percepção } \\
\text { global de } \\
\text { QV }\end{array}$ \\
\hline & & $43,14 \pm 15,04$ & - & & & & & & & \\
\hline & & $652,69 \pm 477,61$ & 0,10 & - & & & & & & \\
\hline & Escolaridade & $3,58 \pm 1,71$ & $-0,25^{* *}$ & $0,36^{* *}$ & - & & & & & \\
\hline \multirow{5}{*}{ 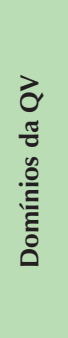 } & $\begin{array}{l}\text { Domínio físico } \\
\text { de QV }\end{array}$ & $68,58 \pm 18,91$ & $-0,22^{* *}$ & $0,22^{* *}$ & $0,13^{*}$ & - & & & & \\
\hline & $\begin{array}{l}\text { Domínio } \\
\text { psicológico de QV }\end{array}$ & $74,88 \pm 15,71$ & $-0,12^{*}$ & $0,14^{*}$ & 0,07 & $0,55^{* *}$ & - & & & \\
\hline & $\begin{array}{l}\text { Domínio social } \\
\text { de QV }\end{array}$ & $65,69 \pm 22,98$ & $-0,15^{* *}$ & $0,16^{* *}$ & $0,15^{* *}$ & $0,34^{* *}$ & $0,51 * *$ & - & & \\
\hline & $\begin{array}{l}\text { Domínio ambiental } \\
\text { de QV }\end{array}$ & $57,96 \pm 17,53$ & 0,007 & $0,32^{* *}$ & $0,19 * *$ & $0,47^{* *}$ & $0,52^{* *}$ & $0,40^{* *}$ & - & \\
\hline & $\begin{array}{l}\text { Percepção global } \\
\text { de QV }\end{array}$ & $60,12 \pm 22,49$ & $-0,11^{*}$ & $0,19 * *$ & 0,06 & $0,55^{* *}$ & $0,54^{* *}$ & $0,39 * *$ & $0,53^{* *}$ & - \\
\hline
\end{tabular}

QV - qualidade de vida; M - média; DP - desvio padrão. $* p<0,05 ; * * p<0,01$. 
de escolaridade descritos na Tabela 1 foram pontuados de 1 (sem escolaridade) a 7 (ensino superior completo). Percebem-se correlações fracas $(r \leq 0,40$ ou $r \leq-0,40)$, porém significativas entre as variáveis idade, renda e escolaridade e entre elas e os domínios de QV. Verifica-se correlação negativa entre idade e escolaridade e correlação positiva entre escolaridade e renda.

A idade apresentou correlação negativa com todos os domínios de QV, exceto ambiental. A renda apresentou correlação positiva com todos os domínios de QV e a escolaridade apresentou correlação positiva com os domínios físico, social e ambiental. Correlações positivas, em sua maior parte moderadas $(0,40<r \leq 0,70)$, foram verificadas entre os domínios de QV e entre eles e a percepção global de QV.

\section{DISCUSSÃO}

A maior parte dos participantes possuía baixa escolaridade. Apenas cerca de um terço deles (32,66\%) tinha concluído o que é considerado no Brasil como Educação Básica (ao menos o Ensino Médio completo). Segundo o Atlas do Desenvolvimento Humano no Brasil ${ }^{15}$, esse índice é de 44,91\% para os ocupados com 18 anos ou mais. A correlação negativa encontrada entre idade e escolaridade é um indicador local da tendência de as gerações mais jovens de homens apresentarem maior escolaridade.

Os usuários, em sua maioria, declararam-se casados ou em união estável $(71,00 \%)$ e a grande maioria possuía filhos (79,00\%), o que dá a dimensão da importância da temática da paternidade em meio a usuários que frequentam a Unidade de Saúde referida. Piccinini et al. ${ }^{16}$ chamam a atenção para a necessidade de atendimentos de pré-natal e pós-natal dirigidos aos homens pais como forma de diminuir a ansiedade e facilitar o envolvimento com os filhos.

A maior parte dos usuários $(79,33 \%)$ não possuía plano privado de saúde, o que reforça a importância dos serviços públicos do SUS para essa população. Tal como na pesquisa de Azevedo et al.7, os participantes apresentaram em geral renda condizente com a "Classe C". Assim como foi constatado pelo Instituto de Pesquisa Econômica Aplicada ${ }^{17}$ para a população brasileira, observou-se correlação positiva entre escolaridade e renda dentre os participantes desta pesquisa.

Apesar de o Brasil apresentar população de maioria católica (64,6\%), segundo o Censo IBGE 2010 ${ }^{18}$, destaca-se que, dentre os participantes com religião, a religião evangélica foi a mais frequente $(41,66 \%)$. Isso pode estar relacionado à predominância que as religiões evangélicas pentecostais e neopentecostais construíram nas últimas décadas do século XX junto às classes populares ${ }^{19}$.
Quanto aos escores de QV avaliados com o Whoqol-Bref, notou-se que o domínio psicológico registrou a maior média, enquanto o domínio ambiental, a menor. É possível comparar esses escores com aqueles de participantes do sexo masculino do estudo de Pucci et al. ${ }^{20}$, que utilizou o mesmo instrumento, aplicado a 540 homens adultos, da população geral, habitantes de Curitiba, a maior parte de classe média ou alta.

Destaca-se que os escores dos domínios físico $(68,58 \pm 18,91)$ e psicológico $(74,88 \pm 15,71)$ foram maiores na presente pesquisa, mesmo se comparados com os resultados obtidos por Pucci et al. ${ }^{20}$ junto a homens da população geral que pertenciam principalmente a classes sociais mais abastadas (respectivamente $56,2 \pm 9,5$ e $65,4 \pm 9,4$ ). Por outro lado, os domínios social e ambiental apresentaram escores mais baixos na comparação $(65,69 \pm 22,98$ para o domínio social e 57,96 $\pm 17,53$ para o domínio ambiental, na presente pesquisa versus respectivamente $74,5 \pm 14,2$ e $65,5 \pm 10,9$, no estudo de Pucci et al. ${ }^{20}$ ).

Múltiplas hipóteses explicativas podem ser levantadas. É possível que homens de classe popular percebam precariedades em seu contexto social e ambiental (apoio social, trabalho, bairro, transporte, etc.) e que elaborem crenças positivas quanto a seus atributos físicos e psicológicos como estratégias individuais e coletivas de enfrentamento das referidas precariedades. A verificação de hipóteses como essa pode ser tarefa de estudos ulteriores.

Assim como em outras pesquisas $6,7,8,9,10,21$, encontrou-se associação positiva entre a $\mathrm{QV}$, a renda e a escolaridade dos participantes, e associação negativa entre QV e idade. Em estudo sobre a QV da população adulta brasileira, utilizando o questionário SF-8, Campolina et al. ${ }^{21}$ encontraram essas mesmas associações. Blay \& Marchesoni ${ }^{6}$ argumentam que maior renda permite acesso a mais recursos e a contextos ambientais mais seguros, o que tem efeitos na saúde e na percepção da QV. É preciso considerar ainda que, na sociedade de consumo, a aceitação social e a construção de autoimagem positiva estão associadas à posse de recursos financeiros e à possibilidade do consumo, inclusive de bens e serviços relacionados à saúde ${ }^{22,23}$.

As diversas transformações físicas, psicológicas e sociais que acompanham o envelhecimento, bem como as atitudes negativas difundidas nas representações sociais sobre os idosos, que incluem elementos como "inutilidade", "perda" e "declínio"24,25, parecem se refletir na percepção dos usuários mais velhos quanto à satisfação com a vida. Um dos aspectos negativos da velhice é a perda do trabalho e do consequente lugar social, contribuindo para o isolamento e a diminuição da autoestima ${ }^{24,25}$. Tendo em vista o lugar central do trabalho para a identidade masculina ${ }^{12}$, é razoável supor que essa perda gere ainda mais impacto para os homens. 
Pesquisas sobre gênero atestaram a centralidade do trabalho pago para a construção da identidade masculina. Trabalhar é a confirmação da virilidade, da potência física, é o suporte para sentimentos de autoestima e autoeficácia e é origem privilegiada de laços sociais ${ }^{12}$. Esse fator, de ordem psicossocial, pode estar relacionado às diferenças encontradas, nesta pesquisa, na percepção de QV entre homens que estavam trabalhando e aqueles que não estavam.

Verificou-se que, mesmo controlando as covariáveis idade e renda, havia diferenças significativas na percepção do domínio físico de QV. É possível que, dentre aqueles que não estavam trabalhando, houvesse certo número de homens com morbidades incapacitantes, que os tenham levado a se afastar do trabalho e que ajudem a explicar a diferença na percepção sobre o domínio físico.

Esses dados e discussões reforçam as recomendações de considerar, no âmbito da APS, determinantes culturais e psicossociais do processo de saúde e doença em homens $s^{11,12,13}$ e de propor estratégias de cuidado individuais e coletivas condizentes. Dentre essas estratégias, destacase que os Grupos de Promoção de Saúde ${ }^{26,27,28}$ podem ser particularmente interessantes.

Grupos direcionados à população masculina, especialmente aos usuários mais idosos, aposentados, afastados do trabalho e/ou portadores de morbidades crônicas podem contribuir para questionar estereótipos, para construir redes sociais e de apoio; promover vínculos com os serviços de saúde e com os profissionais e a adesão a tratamentos; contribuir para a reflexão sobre a trajetória de vida e sobre os projetos de futuro; promover autocuidado, autonomia e qualidade de vida $26,27,28$.

Como considerações finais, cabe assinalar algumas limitações desta pesquisa. A amostra foi obtida por conveniência e apenas com homens que se dirigiram à Unidade de Saúde. Os dados foram coletados em apenas uma USF do município de Vitória, ES. Não foram considerados os diagnósticos dos usuários. Estudos ulteriores poderão angariar informações sobre a QV de homens que não frequentam a USF e/ou que pertençam a estratos de renda diferentes, bem como associações entre morbidades e percepção de qualidade de vida no caso dos usuários do sexo masculino.

A pesquisa realizada preocupou-se especificamente com o campo da Saúde do Homem. Coletou e discutiu informações sociodemográficas e sobre QV de usuários homens que frequentavam uma Unidade de Saúde. Verificou associações entre a idade desses homens, sua escolaridade e sua renda, bem como o fato de estar ou não trabalhando com a QV avaliada com auxílio do Whoqol-Bref. A discussão levou à interpretação desses dados considerando fenômenos psicossociais: estereótipos tradicionais da masculinidade e representações sociais do envelhecimento. Tendo em vista os dados e análises, sugeriu-se o fortalecimento dos Grupos de Promoção da Saúde direcionado aos homens, em âmbito de Atenção Primária à Saúde.

\section{REFERÊNCIAS}

1. Ministério da Saúde (BR). Política Nacional de Atenção Básica. Brasília: Ministério da Saúde; 2012

2. Fertonani HP, Pires DEP, Biff D, Scherer MDA. Modelo assistencial em saúde: conceitos e desafios para a atenção básica brasileira. Ciênc Saúde Coletiva. 2015;20:1869-78. http://dx.doi.org/10.1590/ 1413-81232015206.13272014

3. Seidl EM, Zannon CMLC. Qualidade de vida e saúde: aspectos conceituais e metodológicos. Cad Saúde Pública. 2004;20:580-88. http://dx.doi.org/10.1590/S0102-311X2004000200027

4. Whoqol Group. The World Health Organization Quality of Life Assessment (WHOQOL): position paper from the World Health Organization. Soc Sci Med. 1995;41(10):1403-9. http://dx.doi. org/10.1016/0277-9536(95)00112-K

5. Fleck MPA, Louzada S, Xavier M, Chachamovich E, Vieira G, Santos L, Pinzon V. Aplicação da versão em português do instrumento abreviado de avaliação da qualidade de vida "WHOQOL-bref. Rev Saúde Pública. 2000;34:178-83. http://dx.doi.org/10.1590/S003489102000000200012

6. Blay SL, Marchesoni MSM. Association among physical, psychiatric and socioeconomic conditions and WHOQOL-Bref scores. Cad Saúde Pública. 2011;27:677-86.

7. Azevedo ALS, Silva RA, Tomasi E, Quevedo LA. Doenças crônicas e qualidade de vida na atenção primária à saúde. Cad Saúde Pública. 2013;29:1774-82. http://dx.doi.org/10.1590/S0102311X2013001300017

8. Guedes KD, Guedes HM. Qualidade de vida do paciente portador de insuficiência renal crônica. Ciênc. Saúde (Porto Alegre). 2012;5(1):48-53.

9. Lara CR, Santos FAOG, Silva TJ, Camelier FWR. Qualidade de vida de pacientes renais crônicos submetidos à fisioterapia na hemodiálise. Ciênc. Saúde (Porto Alegre). 2013;6(3):163-71.

10. Alexandre TS, Cordeiro RC, Ramos LR. Factors associated to quality of life in active elderly. Rev Saúde Pública. 2009;43:613-21. http:// dx.doi.org/10.1590/S0034-89102009005000030

11. Ministério da Saúde (BR). Política Nacional de Atenção Integral à Saúde do Homem. Brasília: Ministério da Saúde; 2008.

12. Courtenay, WH. Engendering health: A social constructionist examination of men's health beliefs and behaviors. Psychol Men Masc. 2000;1:4-15. http://dx.doi.org/10.1037/1524-9220.1.1.4

13. Schraiber LB, Figueiredo WS, Gomes R, Couto MT, Pinheiro TF, Machin R, Silva GSN, Valença O. Necessidades de saúde e masculinidades: atenção primária no cuidado aos homens. Cad Saúde Pública. 2010;26:961-70. http://dx.doi.org/10.1590/S0102311X2010000500018

14. Associação Brasileira de Empresas de Pesquisa. Critério de classificação econômica Brasil [Internet]. [acesso em 2015 jul. 21]. Disponível em: http://www.abep.org/criterio-brasil 
15. Programa das Nações Unidas para o Desenvolvimento, Instituto de Pesquisa Econômica Aplicada, Fundação João Pinheiro. Atlas do Desenvolvimento Humano no Brasil [Internet]. [acesso em 2015 jul. 22]. Disponível em: http://www.atlasbrasil.org.br/2013/pt/

16. Piccinini CA, Silva MR, Gonçalves TR, Lopes RCS, Tudge J. Envolvimento paterno aos três meses de vida do bebê. Psic Teor Pesq. 2012; 28:303-14. http://dx.doi.org/10.1590/S0102-37722012000300006

17. Instituto de Pesquisa Econômica Aplicada. Gastos com a política social: alavanca para o crescimento com distribuição de renda. Comunicado [Internet]. 2011(75). [acesso em 2015 jul. 22]. Disponível em: http://www.ipea.gov.br/agencia/images/stories/PDFs/ comunicado/110203_comunicadoipea75_apresentacao.pdf

18. Instituto Brasileiro de Geografia e Estatística. Censo Demográfico 2010. Rio de Janeiro: IBGE; 2012.

19. Mariano, R. Expansão pentecostal no Brasil: o caso da Igreja Universal. Estud Av. 2004;18:121-38. http://dx.doi.org/10.1590/ S0103-40142004000300010

20. Pucci G, Reis RS, Rech CR, Hallal PC. Quality of life and physical activity among adults: population-based study in Brazilian adults. Qual Life Res. 2012;21(9):1537-43. http://dx.doi.org/10.1007/ s11136-011-0083-5

21. Campolina AG, Pinheiro MM, Ciconelli RM, Ferraz MB. Quality of life among the Brazilian adult population using the generic SF-8 questionnaire. Cad Saúde Pública. 2011;27:1121-31. http://dx.doi. org/10.1590/S0102-311X2011000600009
22. Bauman Z. O mal-estar da pós-modernidade. Rio de Janeiro: Jorge Zahar; 1998.

23. Caron E, Lefèvre F, Lefèvre AMC. Afinal, somos ou não somos uma sociedade de consumo? Consequências para a saúde. Ciênc Saúde Coletiva. 2015;20:145-53. http://dx.doi.org/10.1590/141381232014201.18812013

24. Veloz MCT, Nascimento-Schulze CM, Camargo BV. Representações sociais do envelhecimento. Psicol Reflex Crít. 1999; 12:479-501. http://dx.doi.org/10.1590/S0102-797219990002 00015

25. Guerra ACLC, Caldas CP. Dificuldades e recompensas no processo de envelhecimento: a percepção do sujeito idoso. Ciênc Saúde Coletiva. 2010;15:2031-40. http://dx.doi.org/10.1590/S141381232010000600031

26. Tahan-Santos E, Cardoso CL. Experiências de participantes em um Grupo de Promoção de Saúde na Estratégia Saúde da Família. Psico. 2008;39:410-17.

27. Tahan J, Carvalho ACD. Reflexões de idosos participantes de grupos de promoção de saúde acerca do envelhecimento e da qualidade de vida. Saúde Soc. 2010;19:878-88. http://dx.doi.org/10.1590/ S0104-12902010000400014

28. Souza LGS. Profissionais de Saúde da Família e representações sociais do alcoolismo [Tese de Doutorado]. Vitória (ES): Universidade Federal do Espírito Santo; 2012. 